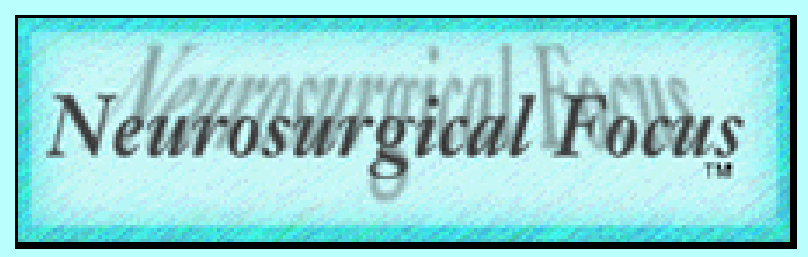

\title{
Commentary from Edward R. Laws, Jr., M.D.
}

From a technical standpoint, there are about as many was to perform a carpal tunnel release as there are to deal with a herniated lumbar disc. Each of the methods has its own champions, often very vocal ones who try to discredit other methods of dealing with the problem. Most experienced neurosurgeons are quite happy with the standard open carpal tunnel release, which uses microsurgical principles. The variations on this technique, the use of a tourniquet or not, the type of incision, and the use of adjunctive steroids, have all been the subject of modest controversy, but each surgeon seems to work out a methodology that is effective in his or her hands.

Certainly having one or another of the endoscopic techniques available for patients is helpful. It is clear that the outcomes with these techniques can be excellent, and in my own experience, they have been ideal for patients who are confined to wheelchairs or need to use crutches and for patients, such as surgeons, who want to get back to work with their hands as rapidly as possible. Which endoscopic technique to use certainly has not been settled; each has its own advantages and disadvantages. When asked when an open carpal tunnel release is preferable to one of the closed techniques, my opinion is that if the patient shows evidence of severe median nerve compression with atrophy and weakness of abductor pollicis brevis, an indirect technique is not as reliable in reversing the symptoms and signs as an open technique in which the median nerve is visualized and can be thoroughly decompressed, removing all overlying thickened ligament. I also tend to avoid the endoscopic method in patients who are obese because perfect performance of the tourniquet is necessary to prevent venous blood from obscuring the endoscopic operative field. Open techniques are preferrable for patients with contracture of the palmar fascia.

Controversies in the management of median thenar neuropathy will continue, but it is fascinating to see how advances in surgical technology result in innovative approaches that ultimately benefit our patients. 